\title{
Inhibition of Neuroblastoma Cell Phosphatidylinositol 3-Kinase by CDP-Diacylglycerol and Phosphatidate
}

\author{
Yaakov Lavie and Bernard W. Agranoff \\ Neuroscience Laboratory, University of Michigan, Ann Arbor, Michigan, U.S.A.
}

\begin{abstract}
Phosphatidylinositol (PI) 3-kinase is activated by a variety of agents, including various growth factors, and has been proposed to play a role in initiation of cell growth, proliferation, and differentiation. We here investigate the effect of various membrane lipids on PI 3-kinase immunopurified from human SH-SY5Y neuroblastoma cells. CDP-diacylglycerol (CDP-DAG) inhibited PI 3-kinase activity with an $\mathrm{IC}_{50}$ of $6 \mu \mathrm{M}$. Phosphatidate (PA) was also inhibitory $\left(\mathrm{IC}_{50}=38 \mu \mathrm{M}\right)$ as was lysophosphatidate. Neither DAG nor any of the other phospholipids examined affected PI 3-kinase activity. The results offer the possibility that CDP-DAG or PA at critical membrane sites may exert functionally significant metabolic regulation at the point of convergence of the PI 3-kinase-directed and the $\mathrm{PI}$ 4-kinase-directed phosphoinositide signal transduction pathways. Key Words: Phosphatidylinositol 3-kinase-CDP-diacylglycerol-Lysophosphatidate-Phosphatidate-SH-SY5Y neuroblastoma cells. J. Neurochem. 66, 811-816 (1996).
\end{abstract}

The SH-SY5Y human neuroblastoma cell line (Pahlman et al., 1990) has proven useful for studying the ligand-stimulated receptor-mediated cleavage of phosphatidylinositol 4,5-bisphosphate $\left[\operatorname{Ptd} \operatorname{Ins}(4,5) \mathbf{P}_{2}\right.$; $\mathrm{PI}(4,5) \mathrm{P}_{2}$ ] into the two chemical second messengers diacylglycerol (DAG) and D-myo-inositol 1,4,5-trisphosphate $\left[\mathrm{I}(1,4,5) \mathrm{P}_{3}\right]$ (Fisher et al., 1992). This subclone is enriched in muscarinic cholinergic receptors and has served as a model system for studies on physiological regulation of the phosphoinositide signal transduction system and of intervention by neuropharmacological agents (Gray et al., 1994). Resynthesis of $\mathrm{PI}(4,5) \mathrm{P}_{2}$ proceeds via phosphorylation of DAG by DAG-kinase and conversion of the resulting phosphatidate (PtdOH; PA) to cytidine diphosphodiacylglycerol (CDP-DAG), which serves, in turn, together with inositol, as a cosubstrate of PI synthase. For the newly formed phosphatidylinositol (PI) to reenter the signal transduction pathway requires that it be phosphorylated sequentially, first at the 4 ' position of inositol, to form phosphatidylinositol 4-phosphate (PI4P), then at the $5^{\prime}$ position to form $\mathrm{PI}(4,5) \mathrm{P}_{2}$. Over the past few years, an alternate fate of PI has been described in which PI 3-kinase acts on PI to produce phosphatidylinositol 3-phosphate (PI3P) (Whitman et al., 1988; Carpenter and Cantley, 1990; Panayotou and Waterfield, 1992; Parker and Waterfield, 1992; Carter and Downes, 1993; Stephens et al., 1993). This isomeric phosphatidylinositol phosphate (PIP), as well as phosphatidylinositol 3,4-bisphosphate $\left[\mathrm{PI}(3,4) \mathrm{P}_{2}\right]$ and phosphatidylinositol 3,4,5-trisphosphate $\left[\mathrm{PI}(3,4,5) \mathrm{P}_{3}\right]$, termed collectively the ' 3 '-phosphoinositides,' have been identified in various tissues and cultured cells in association with growth and differentiation, e.g., after the addition to media of various growth factors (Parker and Waterfield, 1992). There is evidence that intact 3'-phosphoinositides are chemical messengers (Nakanishi et al., 1993; Stephens et al., 1993; Toker et al., 1994), although it remains possible that there are as yet unidentified cleavage products that mediate signal transduction via this pathway. Although it is likely that some 3-phosphoesterification occurs directly on PI4P and on $\mathrm{PI}(4,5) \mathrm{P}_{2}$, the phosphorylation of PI by either PI 3- or 4-kinase would appear to constitute a metabolic branch point, the regulation of which could affect either or both of the pathways. We have established the presence of PI 3-kinase activity in SH-SY5Y cells, as well as its modification by CDP-DAG and by $\mathrm{PA}$, and raise the possibility that regulatory feedback occurs in inositol lipid-mediated signal transduction.

Received May 8, 1995: final revised manuscript received September 12, 1995; accepted September 14, 1995.

Address correspondence and reprint requests to Dr. B. W. Agranoff at Neuroscience Laboratory, University of Michigan, $1103 \mathrm{E}$. Huron, Ann Arbor, MI 48104-1687, U.S.A.

Abbreviations used: CDP-DAG, cytidine diphosphodiacylglycerol; CDS, CDP-DAG synthase; DAG, diacylglycerol: DMEM, Dulbecco's modified Eagle's medium; ECL, enhanced chemiluminescence; FBS, fetal bovine serum; LPA, lysophosphatidate; NP-40, Nonidet P-40; PA, phosphatidate; PBS, phosphate-buffered saline; PC, phosphatidylcholine; PI, phosphatidylinositol; PIP. phosphatidylinositol phosphate; PI3P, phosphatidylinositol 3-phosphate; PI4P, phosphatidylinositol 4-phosphate; $\mathrm{PI}(4,5) \mathrm{P}_{2}$, phosphatidyl 4,5-bisphosphate; SDS-PAGE. sodium dodecyl sulfate--polyacrylamide gel electrophoresis; WT, wortmannin. 


\section{EXPERIMENTAL PROCEDURES}

\section{Materials}

ATP, phospholipids (unless otherwise specified), CMP, CDP, CTP, CDP-glycerol, and bovine serum albumin were purchased from Sigma Chemical (St. Louis, MO, U.S.A.). Stearoylarachidonoyl-CDP-DAG was a generous gift of A. K. Hajra. CDP-DAG (from egg lecithin) was from Serdary Research (London, Ontario, Canada). Cardiolipin was the product of Avanti Polar Lipids (Alabaster, AL, U.S.A.). $\left[\gamma-{ }^{32} \mathrm{P}\right] \mathrm{ATP}(3,000 \mathrm{Ci} / \mathrm{mmol})$ was from Du Pont-NEN (Wilmington, DE, U.S.A.). Silica gel 60 TLC plates were from Merck (Darmstadt, Germany). Dulbecco's modified Eagle's medium (DMEM), fetal bovine serum (FBS), and protein A-agarose were purchased from GibcoBRL (Gaithcrsburg, MD, U.S.A.) . Anti-p85 antiserum was from Upstate Biotechnology Incorporated (Lake Placid, NY, U.S.A.). SHSY5Y cells (60-80 passages) were grown to confluency and treated as described herein.

\section{Preparation of PI 3-kinase}

PI 3-kinase was prepared from confluent SH-SY5Y cells grown in DMEM supplemented with $10 \% \mathrm{FBS}$ at $37^{\circ} \mathrm{C}$ in a humidified atmosphere of $10 \% \mathrm{CO}_{2}$. Cells, grown in 150$\mathrm{mm}$ culture dishes, were washed twice with $15 \mathrm{ml}$ of phosphate-buffered saline (PBS), pH 7.4, aspirated, and lysed with $2 \mathrm{ml} /$ dish of lysis buffer containing $137 \mathrm{~m} M \mathrm{NaCl}, 20$ $\mathrm{m} M$ Tris-HCl (pH 8.0), $1 \mathrm{~m} M$ EDTA, $1 \mathrm{~m} M \mathrm{MgCl}_{2}, 1$ $\mathrm{m} M \mathrm{CaCl}_{2}, 10 \%$ glycerol, $1 \%$ Nonidet P-40 (NP-40), 10 $\mu \mathrm{g} / \mathrm{ml}$ leupeptin, $2 \mu \mathrm{g} / \mathrm{ml}$ aprotinin, $1 \mathrm{~m} M$ phenylmethylsulfonyl fluoride, and $1 \mathrm{~m} M$ sodium orthovanadate. The cells were scraped free from the dishes and centrifuged at 6,000 $g$ for $10 \mathrm{~min}$. The resulting supernatant was incubated in 50ml conical plastic tissue-culture tubes with shaking overnight with $4 \mu \mathrm{g}$ of anti-p85 antiserum per milligram of protein, after which protein A-agarose beads were added for $2 \mathrm{~h}$ with mixing. Immune complexes bound to the beads were washed three times with PBS containing 1\% NP-40, twice with PBS containing $0.5 \mathrm{M} \mathrm{LiCl}$ and $0.1 M$ Tris- $\mathrm{HCl}(\mathrm{pH} 7.5)$, and twice with $0.1 \mathrm{M} \mathrm{NaCl}$ containing $1 \mathrm{~m} M$ EDTA and $10 \mathrm{~m} M$ Tris- $\mathrm{HCl}(\mathrm{pH} 7.5)$.

\section{PI 3-kinase assay}

The reaction mixture for measuring PI 3-kinase activity contained $50 \mu \mathrm{l}$ of the immunoprecipitated enzyme preparation (representing enzyme from $5 \times 10^{\prime \prime}$ cells) and $50 \mu \mathrm{l}$ of PI micelles that were prepared by sonicating $800 \mu \mathrm{g}$ of PI ( Sigma, from bovine liver) in $1 \mathrm{ml}$ of $20 \mathrm{~m} M$ HEPES buffer ( $\mathrm{pH} 7.6$ ) containing $1 \mathrm{~m} M$ EDTA for $45 \mathrm{~s}$ (Kontes sonicator with a microlip probe). Fifty microliters of HEPES buffer or various sonicated lipids to be tested were added at this point. The mixture was preincubated for $15 \mathrm{~min}$ at room temperature, and the reaction was started by addition of 50 $\mu \mathrm{l}$ of $20 \mathrm{mM} \mathrm{MgCl} \mathrm{M}_{2}$ containing $150 \mu M\left[\gamma^{-}{ }^{32} \mathrm{P}\right] \mathrm{ATP}$ (20 $\mu \mathrm{Ci}$ per assay). The reaction was terminated after $10 \mathrm{~min}$ by the addition of $200 \mu \mathrm{l}$ of $1 \mathrm{M}$ methanolic $\mathrm{HCl}$ followed by $200 \mu \mathrm{l}$ of chloroform. The resulting organic phase was dried, resuspended in $25 \mu \mathrm{l}$ of chloroform/methanol ( $1: 1$ ), and spotted on a TLC plate that was precoated with $1 \%$ potassium oxalate. The plate was developed in chloroform/ methanol $/ \mathrm{H}_{2} \mathrm{O} / 7.7 \mathrm{M}$ ammonium hydroxide (60:47:1 I.3:2) (Serunian et al., 1991). ${ }^{32}$ P-labeled PI3P was visualized by autoradiography and compared with an iodine-stained authentic PI4P standard. Radioactivity in PI3P was quantitated by liquid scintillation spectrometry.
A $\quad$ Mr $\left(\times 10^{-3}\right)$
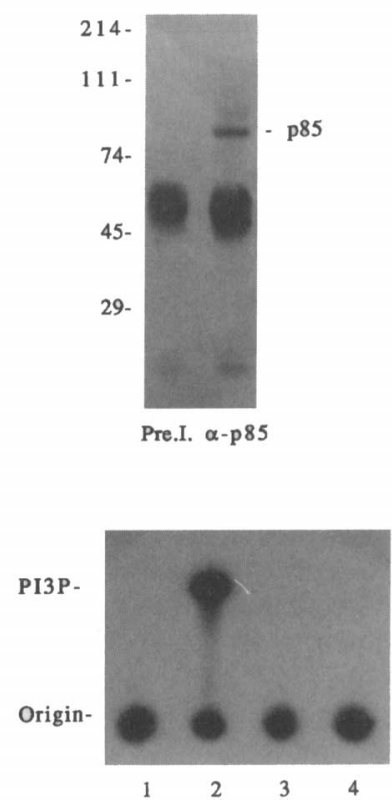

FIG. 1. Immunoblot and enzymatic activity of PI 3-kinase isolated from SH-SY5Y cells with p85 anti-sera. A: PI 3-kinase was immunoprecipitated from SH-SY5Y cells, as described in Experimental Procedures, and immunoblotted after SDS-PAGE, using either preimmune rabbit anti-serum (Pre.l.) or anti-p85 anti-serum ( $\alpha$-p85). The prominent spots above the 45-kDa mark represent IgG. B: The immunoprecipitates were assayed for PI 3kinase activity as described in the text. Lane 1, preimmune serum immunoprecipitates; lane 2, $\alpha$-p85 immunoprecipitates; lane 3 , $\alpha$-p85 + 0.5\% NP-40; lane $4, \alpha-\mathrm{p} 85+50 \mathrm{n} M \mathrm{WT}$. The data shown are representative of three independent experiments, which gave similar results.

\section{Immunoblotting of the p85 subunit of PI 3-kinase}

Cells were lysed, followed by immunoprecipitation with anti-p85 antiserum as described. After washing, immune complexes were resolved on $10 \%$ sodium dodecyl sulfatepolyacrylamide gel electrophoresis (SDS-PAGE). Separated proteins were transferred to nitrocellulose paper and analyzed for the presence of $\mathrm{p} 85$ by protein immunoblot using anti-p85 antiserum. Bound antibodies were visualized by an enhanced chemiluminescence (ECL) detection system, using horseradish peroxidase conjugated to anti-rabbit $\operatorname{lgG}$ as the secondary antibody (Amersham, Arlington, Heights, IL. U.S.A.).

\section{RESULTS}

\section{Assay of PI 3-kinase}

Human SH-SY5Y neuroblastoma cells were found to be a ready source of PI 3-kinase. The presence of the $\mathrm{p} 85$ protein in anti-p85 immunoprecipitates from these cells was confirmed by western blotting with the same antiserum and detection by ECL (Fig. 1A). A major band at the $85-\mathrm{kDa}$ region was visualized in the anti-p85 antiserum immunoprecipitates and not in those prepared with preimmune rabbit serum. PI is the 


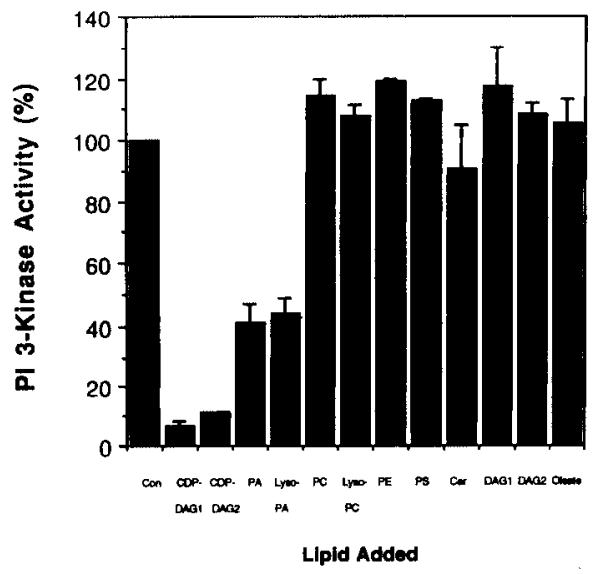

FIG. 2. Effect of various lipids on PI 3-kinase activity. PI 3-kinase activity was measured under standard assay conditions with 200 $\mu M \mathrm{Pl}$ and $50 \mu \mathrm{M}$ labeled ATP in the absence and presence of $100 \mu M$ concentrations of the indicated lipids. The data shown are representative of three independent experiments that gave similar results. Control value of $\left[{ }^{32} \mathrm{P}\right] \mathrm{PI} P \mathrm{P}$ was $6,298 \pm 518 \mathrm{cpm}$. Con, control; CDP-DAG1, egg PC-derived CDP-DAG; CDPDAG2, stearoylarachidonoyl-CDP-DAG; PE, phosphatidylethanolamine; PS, phosphatidylserine; Car, cardiolipin; DAG1, dioleoylacylglycerol; DAG2, stearoylarachidonoyl-DAG.

major intracellular substrate for PI 3-kinase (Berggren et al., 1993) and was therefore used for these studies. Optimum incubation conditions were those described by Serunian et al. (1991); phosphorylation in SHSY5Y cells was found to be linear with time and enzyme concentration (not shown). As indicated in Fig. 1B, PI phosphorylation to PIP was detected in the antip85 immunoprecipitates but not in preimmune rabbit serum immunoprecipitates. To examine the possible presence of PI 4-kinase activity in the preparations, we performed the phosphorylation reaction in the presence of NP-40, a detergent known to inhibit PI 3kinase activity and to stimulate PI 4-kinase activity (Carpenter and Cantley, 1990). Addition of 0.5\% NP40 to the reaction mixture inhibited the formation of ${ }^{32}$ P-labeled lipid completely, indicating the absence of detectable PI 4-kinase activity in the preparation. Furthermore, we found that wortmannin (WT) inhibited the phosphorylation of PI in our preparation completely (Fig. 1B). WT is found in a variety of fungi and has been shown to selectively block PI 3-kinase activity in vitro and in vivo by binding covalently to the 110-kDa subunit of PI 3-kinase (Powis et al., 1994).

\section{Effect of various lipids on PI 3-kinase activity}

Membrane-bound enzymes, especially those that mediate membrane lipid metabolism are generally known to be responsive to their lipid environment (Hjelmstad and Bell, 1991). We therefore examined the effect of various membrane lipids on PI 3-kinase activity in vitro at saturating concentrations of $\mathrm{Mg}^{2+}$, ATP, and PI. As shown in Fig. 2, egg phosphatidylcho- line (PC)-derived CDP-DAG and stearoyl-arachidonoyl-CDP-DAG produced 93 and $88 \%$ inhibition of PI3P formation, respectively. The observed block by CDP-DAG, therefore, does not appear to be highly selective with regard to the fatty acid composition of the molecule. CDP-glycerol, the deacylated form of CDP-DAG, had no effect on PI 3-kinase activity (see Table 1), indicating that fatty acyl substituents are required for the inhibitory effect. Lyso-CDP-DAG was not tested. PI 3-kinase activity was also inhibited, albeit to a lesser extent (59\%), by PA. 1-Oleoyl-snglycero-3-P (lyso-PA; LPA) inhibited PI 3-kinase to the same extent as PA $(t=0.4, \mathrm{NS})$, whereas lyso$P C$ was ineffective. None of the other phospholipids examined, i.e., PC, phosphatidylethanolamine, phosphatidylserine, or cardiolipin, significantly affected PI 3-kinase activity, nor did either of two species of DAG used in this study, dioleoylglycerol or stearoylarachidonoyldiacylglycerol (also see Fig. 3B).

\section{Characterization of the inhibitory effect of CDP-DAG on PI 3-kinase}

The concentration dependence of the inhibition of PI 3-kinase activity by CDP-DAG and PA is evident in an autoradiogram made after TLC separation of phosphorylated PI lipids from PI 3-kinase assays, which contain the inhibitory lipids (Fig. 3A). The plots in Fig. 3B demonstrate that CDP-DAG and PA inhibit PI 3-kinase in a dose-dependent manner, with estimated $\mathrm{IC}_{50}$ values of 6 and $38 \mu M$, respectively.

Because of the unique structure of CDP-DAG, we investigated the possibility that separate nucleotideand lipid-binding sites, acting together, might explain the liponucleotide's blocking action. By adding various moieties of CDP-DAG alone or together, we established that the intact molecule is required for inhibition.

TABLE 1. The effect of CDP-DAG and related compounds on $I^{32} P J P I 3 P$ formation

\begin{tabular}{lcc}
\hline Lipid added & PI3P lormed $(\mathrm{cpm})$ & \% of inhibition \\
\hline None & $6,759 \pm 583$ & 0 \\
CDP-DAG & $830 \pm 69$ & $88 \pm 8$ \\
PA & $2,430 \pm 137$ & $64 \pm 6$ \\
CMP & $6,408 \pm 231$ & $5 \pm 4$ \\
PA + CMP & $2,651 \pm 302$ & $61 \pm 11$ \\
DAG & $6,709 \pm 159$ & $1 \pm 2$ \\
CDP & $6.883 \pm 531$ & 0 \\
DAG + CDP & $6,847 \pm 885$ & 0 \\
CTP & $5,872 \pm 398$ & $13 \pm 7$ \\
CDP-glycerol & $6,651 \pm 729$ & $2 \pm 11$ \\
\hline
\end{tabular}

PI 3-kinase activity in anti-PI 3-kinase antibody immunoprecipitates from SH-SY5Y cells was measured as the [ $\left.{ }^{32} \mathrm{P}\right] \mathrm{ATP}$-dependent phosphorylation of PI, as described in Experimental Procedures. The indicated compounds were added at a concentration of $100 \mu \mathrm{M}$ for 10 min of incubation. Results are expressed as percentages of inhibition of PI3P formed in the reaction and represent mean $\pm \mathrm{SD}$ (half the range) values of duplicate determinations, and are typical of three experiments. 


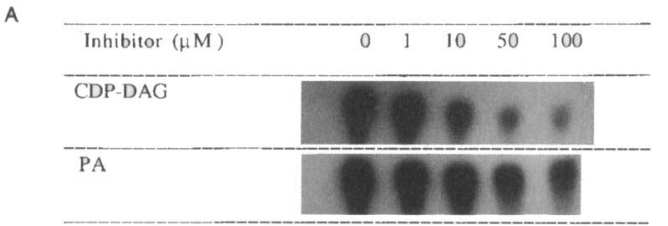

B

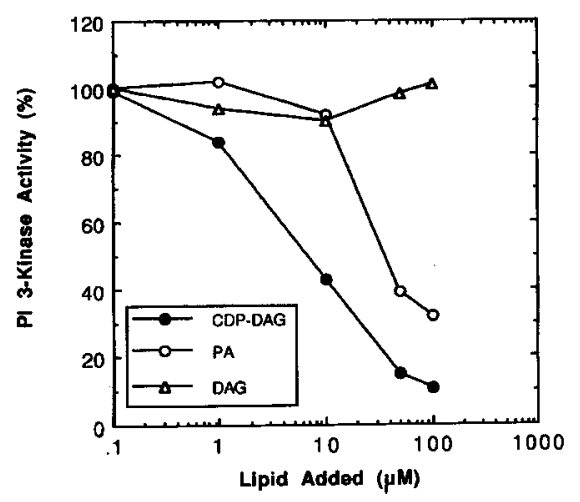

FIG. 3. Inhibition of PI 3-kinase activity by CDP-DAG and PA. $\mathrm{PI}$ 3-kinase was assayed in the presence of the indicated concentrations of egg PC-derived CDP-DAG (closed circles), PA (open circles), or DAG (triangles). The phosphorylated lipid products were separated by TLC and then subjected to autoradiography $(\mathbf{A})$. The corresponding PIP bands were scraped and counted. The PI 3-kinase activity is plotted $(\mathbf{B})$ as the percentage of uninhibited kinase activity $(5,791 \pm 451 \mathrm{cpm} ; 100 \%)$.

Neither cytidine mono-, di-, nor triphosphate altered PI 3-kinase activity (Table 1). Addition of PA and CMP together produced inhibition similar to that caused by PA alone, and the combination of DAG plus CDP had no effect, confirming that the inhibitory effect of CDP-DAG requires the intact liponucleotide molecule. To examine the nature of the inhibition further, we determined whether the effect of CDP-DAG could be reversed by the addition of various concentrations of the substrate PI or ATP. As shown in Table 2, even with concentrations of PI and/or ATP five to 10 times higher than were routinely used, the degree of inhibition was unaffected. Although we did not examine these effects at other CDP-DAG concentrations, it does not appear likely that CDP-DAG is a competitive inhibitor with respect to PI or ATP.

\section{DISCUSSION}

Demonstration of the presence of PI 3-kinase in SHSY5Y neuroblastoma cells and its inhibition by two endogenous classes of lipids was made possible by the availability of a high-affinity anti-p85 (regulatory subunit) antibody that permitted us to immunopurify an enzyme complex that also contained the $110-\mathrm{kDa}$ catalytic subunit of PI 3-kinase. The isolated protein complex phosphorylated PI to a product that migrated on TLC to a region predicted for PIPs. Although PI3P and PI4P are not easily separable, the conclusion that the product was solely PI3P was also supported by the complete block of phosphorylation in the presence of WT, an agent known to block selectively PI 3-kinase but not PI 4-kinase. The result also indicated that there was no detectable activity for a recently described WTinsensitive PI-specific PI kinase (Stephens et al., 1994) in these cells.

Although the inhibitory effect of CDP-DAG does not appear to be restricted to a specific species of the liponucleotide, fatty acylation of the glycerol moiety is essential for the inhibitory action, as is the CDP head group, because CDP-glycerol and DAG are without effect, whereas inhibition, albeit to a lesser extent, is observed with PA. The block by PA is in agreement with a previous report (Carpenter et al., 1990) in which purified PI 3-kinase from rat liver was shown to be inhibited $50 \%$ by $10 \mu M$ PA. LPA was equally effective, as has been observed with other cellular actions of PA and LPA such as their mitogenic actions (Moolenaar, 1995). Recent studies indicate that LPA can activate PI 3-kinase (Kumagai et al., 1993; Moolenaar, 1995). The stimulatory effect is distinct from the present report of an inhibitory action of LPA in that the former is mediated by its receptor and a $\mathrm{G}$ proteinlinked signal transduction cascade, whereas the latter is the direct action of LPA on PI 3-kinase. Inhibition of PI 3-kinase by CDP-DAG has not been reported previously, although its block of PI 4-kinase has been observed in yeast, as discussed herein. Both CDP-DAG and PA are negatively charged phospholipids, and the mechanism of the observed inhibition might therefore be attributed to their negative charge. However, phosphatidylserine and cardiolipin, which are also negatively charged, had no effect on PI 3-kinase activity. Although it remains possible that a negative charge on inhibitory lipids is necessary, it does not appear to be

TABLE 2. Effects of ATP and PI on the inhibitory action of $C D P-D A G$

\begin{tabular}{rcc}
\hline PI $(\mu M)$ & ATP $(\mu M)$ & PI 3-kinase activity $(\%)$ \\
\hline 40 & 50 & $16.1 \pm 4.3$ \\
80 & 50 & $19.1 \pm 3.2$ \\
200 & 50 & $13.0 \pm 1.5$ \\
1,000 & 50 & $15.4 \pm 0.9$ \\
200 & 5 & $17.8 \pm 3.7$ \\
200 & 500 & $8.7 \pm 3.1$ \\
\hline
\end{tabular}

PI 3-kinase activity in anti-PI 3-kinase antibody immunoprecipitates was assayed in the presence of the indicated concentrations of PI, ATP, and $50 \mu M$ CDP-DAG. The assay was started by adding the enzyme, allowing equal opportunity for the inhibitor and the competing substrates to bind. The determined lipid kinase activity is given as a percentage of the uninhibited (minus CDP-DAG) lipid kinase activity $(100 \%)$ for each case. The results represent mean \pm SD values of duplicate determinations and are typical of three experiments. 
sufficient for the inhibitory effect. The loci of CDPDAG and PA binding are unknown but do not appear to be at the ATP- or PI-binding sites. On the basis of findings in the analogous yeast protein VPS34, an inferred lipid-binding site should be considered ( see Stephens et al., 1993).

The relevance of these observations to in vivo conditions remains questionable. CDP-DAG is a quantitatively minor lipid; Thompson and Macdonald (1976) reported the presence of $13.2 \mu \mathrm{mol} C D P-D A G / \mathrm{kg}$ of bovine brain. The present finding of an $\mathrm{IC}_{50}$ of $6 \mu \mathrm{M}$ for inhibition of PI 3-kinase suggests that CDP-DAG could well regulate this enzyme in vivo. Inhibition would be further enhanced under conditions in which intracellular CDP-DAG appears to be elevated. When tissue slices (Godfrey, 1989) or cultured cells (Stubbs and Agranoff, 1993) are pretreated with labeled cytidine, and then with carbachol in the presence or absence of $\mathrm{Li}^{+}$, labeled CDP-DAG is greatly increased, presumably because PI-synthase activity has become substrate limited for free inositol, on the basis of the inhibition of inositol monophosphatase by $\mathrm{Li}^{+}$(Berridge et al., 1989). In the case of SH-SY5Y cells, the increase is seen only if they have been deprived of inositol in the incubation medium as well (Stubbs and Agranoff, 1993). Although CDP-DAG mass has not been measured in rat brain, $\left[{ }^{3} \mathrm{H}\right]$ cytidine-labeling studies in neurotransmitter-stimulated rat brain slices indicate a heterogeneous distribution that is presumed to indicate a broad range of amounts of the liponucleotide among brain cell types as well (Hwang et al., 1990).

It is of interest that the concentration of CDP-DAG in plasma membranes of inositol-starved Inol yeast cells was found to be 12-fold higher than in inositolsupplemented cells, and that this elevation was associated with inhibition of PI 4-kinase (Nickels et al., 1994). It is not yet known whether mammalian PI 4kinase activity is affected by phospholipids. CDPDAG in eukaryotic cells appears to be compartmented intracellulary in distinct mitochondrial and endoplasmic reticulum sites for the formation of cardiolipin and phospoinositides, respectively (Mok et al., 1992). On the basis of the recent cloning of a Drosophila CDPDAG synthase (CDS) gene and identification of a defective mutant (Wu et al., 1995), it appears that cell types may vary in isoforms of CDS and thus could also vary in levels of CDP-DAG under resting and stimulated conditions. Although PA inhibits PI 3-kinase with a somewhat higher $\mathrm{IC}_{50}(38 \mu M)$ than found for CDP-DAG, it should be noted that PA is present in 100-fold higher amounts than CDP-DAG in adult rat brain, and increases with age from $14 \mu M$ in 3day-old rats to $1.3-1.4 \mathrm{~m} M$ in 330-day-old rat brains (Wells and Dittmer, 1967). A similar value for PA was found in bovine brain (Thompson and Macdonald, 1976). We have previously reported that SK-N-SH cells, the parent line of the SH-SY5Y subclone, contain $20 \mathrm{nmol} \mathrm{PA} / \mathrm{mg}$ of protein (Lee et al., 1991), corre- sponding to a calculated concentration of $20 \mu \mathrm{M}$, and that carbachol more than doubles the PA concentration, bringing it well within the range of the in vitro $\mathrm{IC}_{50}$ $(38 \mu M)$ observed in this study. In addition, hormoneinduced generation of PA is reported to occur widely via activation of phospholipase $D$ or the sequential activation of phospholipase $\mathrm{C}$ and DAG kinase (see Liscovitch, 1992). It may then be concluded that unless intracellular compartmentation prevents access, PA likely regulates PI 3-kinase in vivo.

These in vitro studies suggest that in vivo regulation of PI 3-kinase activity by intracellular PA and/or by CDP-DAG may underlie a functional relationship of these metabolic precursors with the PI3P and PI4P inositol lipids.

\section{REFERENCES}

Berggren M. I., Gallegos A., Dressler L. A.. Modest E. J., and Powis G. ( 1993) Inhibition of the signalling enzyme phosphatidylinositol-3-kinase by antitumor ether lipid analogues. Cancer Res. 53, $4297-4302$.

Berridge M. J., Downes C. P.. and Hanley R. M. (1989) Neural and developmental actions of lithium: a unifying hypothesis. Cell 59, 4 l $\mathrm{l}-419$.

Carpenter C. L. and Cantley L. C. ( 1990 ) Phosphoinositide kinases. Biochemistry 29, 11147-11156.

Carpenter L. C., Duckworth B. C., Auger K. R., Cohen B., Schaffhausen B. S., and Cantley L. C. (1990) Purification and characterization of phosphoinositide 3-kinase from rat liver. $/$. Biol. Chem. 265, 19704-19711.

Carter A. N. and Downes C. P. (1993) Signaling by neurotrophic factors: activation of phosphoinositide 3 -kinase by nerve growth factor. Neuroprotocols 3,107-118.

Fisher S. K., Heacock A. M., and Agranoff B. W. (1992) Inositol lipids and signal transduction in the nervous system: an update. I. Neurochem. 58, 18-38.

Godfrey P. P. (1989) Potentiation by lithium of CDP-DAG formation in carbachol-stimulated rat cerebral-cortical slices and its reversal by myo-inositol. Biochem. J. 258, 621-624.

Gray D. W., Challiss R. A. J., and Nahorski S. R. ( 1994 ) Differential effects of lithium on muscarinic cholinoceptor-stimulated CMPphosphatidate accumulation in cerebellar granule cells, CHOM3 cells and SH-SY5Y cells. I. Neurochem. 63, 1354-1360.

Hjelmstad R. H. and Bell R. M. (1991) Molecular insights into enzymes of membrane bilayer assembly. Biochemistry 30, $1731-1740$.

Hwang P. M., Bredt D. S., and Snyder S. H. (1990) Autoradiographic imaging of phosphoinositide turnover in the brain. Science 249, $802-804$.

Kumagai N., Morii N., Fujisawa K., Nemoto N., and Shuh N. (1993) ADP-ribosylation of the $\mathrm{p} 2 \mathrm{I}$ inhibits lysophosphatidic acidinduced protein tyrosine phosphorylation and phosphatidylinositol 3-kinase activation in cultured Swiss $3 T 3$ cells. J. Biol. Chem. 268, 24535-24538.

Lee C. Fisher S. K., Agranoff B. W., and Hajra A. K. (1991) Quantitative analysis of molecular species of diacylglycerol and phosphatidate formed upon muscarinic receptor activation of human SK-N-SH neuroblastoma cels. J. Biol. Chem. 266, $22837-22846$.

Liscovitch M. (1992) Crosstalk among multiple signal-activated phospholipases. Trends Biochem. Sci. 17, 393-399.

Mok A. Y. P., McDougall G. E., and McMurray W. C. (1992) CDPdiacyl glycerol synthesis in rat liver mitochondria. FEBS Lett. $312,236-240$.

Moolenaar H. M. (1995) Lysophosphatidic acid, a multifunctional phospholipid messenger. J. Biol. Chem. 270, 12949-12952. 
Nakanishi H., Kimberly A. B., and Exton J. H. (1993) Activation of the $\zeta$ isozyme of protein kinase $C$ by phosphatidylinositol 3,4,5-trisphosphate. J. Biol. Chem. 268, 13-16.

Nickels J. T., Buxeda R. J., and Carman G. M. (1994) Regulation of phosphatidylinositol 4-kinase from the yeast Saccharomyces cerevisiae by CDP-diacylglycerol. J. Biol. Chem. 269, 1101811024.

Pahlman S., Mamaeva S., Meyerson G., Mattson M. E. K., Bjelfman C. Ortoft E., and Hammerling U. (1990) Human neuroblastoma cells in culture: a model for neuronal cell differentiation and function. Acta Physiol. Scand. Suppl. 592, 25-37.

Panayotou G. and Waterfield M. D. (1992) Phosphatidylinositol 3kinase: a key enzyme in diverse signalling processes. Trends Cell Biol. 2, 358-360.

Parker P. J. and Waterfield M. D. (1992) Phosphatidylinositol 3kinase: a novel effector. Cell Growth Differ. 3, 747-752.

Powis G., Bonjouklian R., Berggren M. M., Gallegos A., Abraham R., Ashendel C., Zalkow L., Matter W. F., Dodge J., Grindey G., and Vlahos C. J. (1994) Wortmannin, a potent and selective inhibitor of phosphatidylinositol 3-kinase. Cancer Res. 54, 2419-2423.

Serunian L. A., Auger K. R., and Cantley L. C. ( 1991 ) Identification and quantification of polyphosphoinositides produced in response to PDGF stimulation. Methods Enzymol. 198, 78-87.

Stephens L. R., Jackson T. R., and Hawkins P. T. (1993) Agoniststimulated synthesis of phosphatidylinositol $(3,4,5)$-trisphos- phate: a new intracellular signalling system? Biochim. Biophys. Acta 1179, 27-75.

Stephens L., Cooke F. T., Walters R., Jackson T., Volina S., Gout I., Waterfield M. D., and Hawkins P. T. ( 1994 ) Characterization of phosphatidylinositol-specific phosphoinositide 3-kinase from mammalian cells. Current Biol. 4, 203-214.

Stubbs E. B. Jr. and Agranoff B. W. (1993) Lithium enhances muscarinic receptor-stimulated CDP-diacylglycerol formation in inositol-depleted SK-N-SH neuroblastoma cells. J. Neurochem. 60, $1292-1299$.

Thompson W. and Macdonald G. (1976) Cytidine diphosphate diglyceride of bovine brain. Eur. J. Biochem. 65, 107-111.

Toker A., Meyer M., Reddy K. K., Falck J. R., Aneja R., Aneja S., Parra A., Burns D. J., Ballas L. M., and Cantley L. C. (1994) Activation of protein kinase $\mathrm{C}$ family members by the nove]

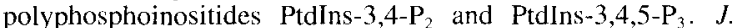
Biol. Chem. 269, 32358-32367.

Wells M. A. and Dittmer J. C. (1967) A comprehensive study of the postnatal changes in the concentration of the lipids of developing rat brain. Biochemistry 6, 3169-3175.

Whitman M., Downes C. P., Keeler M., Keller T., and Cantley L. (1988) Type I phosphatidylinositol kinase makes a novel inositol phospholipid, phosphatidylinositol-3-phosphate. Nature 332, $644-646$.

Wu L., Niemyer B., Colley N., Socolich M., and Zuker C. S. (1995) Regulation of PLC-mediated signalling in vivo by CDP-diacylglycerol synthase. Nature 373, 216-222. 